\title{
Prenatal detection and molecular cytogenetic characterization of $19 q 13.42$ microduplication: three reported cases and literature review
}

Xinyue Zhang ${ }^{1,2}$, Fagui Yue ${ }^{1,2}$, Qingyang Shi ${ }^{1,2}$, Yuting Jiang ${ }^{1,2}$, Jing He ${ }^{1,2}$, Leilei Li $i^{1,2}$ and Ruizhi Liu $u^{1,2^{*}}$ (])

\begin{abstract}
Background: Trisomy $19 q$ is a recognizable syndrome and associated with a wide spectrum of clinical phenotypes in clinic. The purpose of this study was to explore the prenatal phenotypes of 19q13.42 duplication, which was rarely reported in clinic.
\end{abstract}

Case presentation: Three pregnant women presenting diverse indications for prenatal diagnosis accepted amniocentesis: increased nuchal translucency and fetal pyelic separation (case 2) and high risk of maternal serum screening for Down syndrome (case 1 and case 3). Case 1 and case 2 shared similar duplicated locus in the region of 19q13.42, encompassing part NLRP12 gene. The latter inherited the chromosomal duplication from the mother with normal phenotypes. Case 3 carried a $1.445 \mathrm{Mb}$ duplication in the 19q13.42q13.43 region. It was proposed that evolutionary duplication of NLRP12 gene could have a causative role in autoinflammatory diseases development. The genotypephenotype correlation depends mainly on the duplicated size and functional genes involved, which is still yet to be determined. All pregnant women chose to continue the pregnancy and delivered healthy children with no apparent abnormalities.

Conclusions: The 19q13.42 microduplications in our study were the smallest fragments compared to previous literature. Our findings enriched the prenatal phenotypes for this chromosomal microscopic imbalance. It was proposed that long term follow up analysis should be guaranteed till adulthood to determine whether there will be other emerging clinical symptoms and developmental-behavioral disorders for such carriers.

Keywords: 19q13.42 mciroduplication, Chromosomal microarray analysis, Prenatal phenotypes, Follow up

\section{Background}

Chromosomal duplications are regarded to have close association with intellectual disability, growth retardation and other genetic disorders. Trisomy 19q, partial or entire duplication of long arm of chromosome 19, was a rare chromosomal anomaly which was first reported in

*Correspondence: Irz410@126.com

${ }^{1}$ Center for Reproductive Medicine, Center for Prenatal Diagnosis, First Hospital, Jilin University, Changchun 130021, China

Full list of author information is available at the end of the article
1976 [1]. Currently, the incidence rate was not clear in clinic. The clinical features of trisomy 19 described in previously reported patients were various and included growth retardation, developmental delay, intellectual disability, microcephaly, heart malformations, anomalies of the genito-urinary tract and/or the gastrointestinal system and seizures, which usually led to poor prognosis [2-5].

The formation mechanism of trisomy $19 q$ could be due to the presence of a de novo duplication, parental balanced reciprocal translocation, pericentric inversion, or original author(s) and the source, provide a link to the Creative Commons licence, and indicate if changes were made. The images or other third party material in this article are included in the article's Creative Commons licence, unless indicated otherwise in a credit line to the material. If material is not included in the article's Creative Commons licence and your intended use is not permitted by statutory regulation or exceeds the permitted use, you will need to obtain permission directly from the copyright holder. To view a copy of this licence, visit http://creativecommons.org/licenses/by/4.0/. The Creative Commons Public Domain Dedication waiver (http://creativeco mmons.org/publicdomain/zero/1.0/) applies to the data made available in this article, unless otherwise stated in a credit line to the data. 
small supernumerary marker, which made it difficult to define a distinct genotype-phenotype correlation [6-8].

For prenatal cases presenting normal karyotypes, chromosomal submicroscopic imbalances of clinic significance could be diagnosed in approximately $1 \%$ of structurally normal pregnancies and 6\% with structural malformations. Most frequent copy number variants (CNVs) observed in fetuses without structural anomalies included 15q11.2, Xp22.3, Xp21.1, 16p11.2, 1q21.1, 17p12, 16p13.11 and 22q11.21 [9]. To our knowledge, the first case of dup(19q) detected by prenatal diagnosis was reported in 1997 [3]. Herein, we describe three prenatal cases involving 19q13.42 microduplications presenting ultrasound anomalies or not, which are the smallest fragments compared to previous trisomy $19 \mathrm{q}$ cases.

\section{Case presentation}

\section{Participants and clinical data}

Three pregnant women underwent amniocentesis for cytogenetic and chromosomal microarray analysis (CMA) due to various indications for prenatal diagnosis: increased nuchal translucency and fetal pyelic separation (case 2) and high risk of maternal serum screening for Down syndrome (case 1 and case 3). All couples were nonconsanguineous and healthy. No family history of diabetes mellitus or congenital malformations were observed. All pregnant women denied any exposure to alcohol, teratogenic agents, irradiation, or infectious diseases during their pregnancies. This study protocol was approved by the Ethics Committee of the First Hospital of Jilin University (No. 2019-299), and written informed consents were obtained from all couples for publication of this case report and accompanying images.

\section{Methods}

\section{Cytogenetic analysis}

Amniocentesis was performed for karyotyping analysis with informed consent. Cytogenetic studies were performed on metaphases collected from cultured amniotic fluid cells from three pregnant women. Routine chromosome analysis was performed on G-banding techniques at 300-400 banding resolution prepared from the cultured amniotic fluid cells according to standard protocols. Twenty metaphases were analyzed for all samples. The International System for Human Cytogenetic Nomenclature (ISCN 2016) was used to describe the karyotype [10].

\section{Chromosomal microarray analysis}

Genomic DNA was isolated from $10 \mathrm{~mL}$ amniotic fluid cells from all three pregnant women. The CytoScan $750 \mathrm{~K}$ array (Affymetrix, Santa Clara, CA, USA) was applied to detect the known and novel chromosomal CNVs across the entire genome following the manufacturer's protocols and our previous study [11]. The procedures included genomic DNA extraction, digestion and ligation, PCR amplification, PCR product purification, quantification and fragmentation, labeling, array hybridization, washing and scanning. The CNVs detected were totally assessed by comparing them with published literature and the public databases: (1) Database of Genomic Variants (DGV) (http://dgv.tcag.ca/dgv/app/home), (2) DECIPHER (http://decipher.sanger.ac.uk/), (3) ISCA (https:// www.iscaconsortium.org/) and (4) OMIM (http://www. ncbi.nlm.nih.gov/omim). Genomic positions refer to the Human Genome February 2009 assembly (GRCh37/ hg19).

\section{Results}

Case 1

A 30 -year-old, gravida 1, para 0 , abortus 2, pregnant woman underwent amniocentesis for cytogenetic analysis and CMA detection due to the high risk of maternal serum screening for Down syndrome. No ultrasound findings were observed at 18 weeks of gestation. G-banding analysis showed that the karyotype of the fetus was $46, \mathrm{XY}$, but CMA revealed a $147 \mathrm{~kb}$ duplication in the region of 19q13.42. In order to identify whether the microduplication was de novo or parentally inherited, the couple accepted CMA after informed consents. It turned out that case 1 inherited the 19q microduplication from the mother with normal phenotypes. The couple chose to continue the pregnancy according to genetic counseling and delivered a male infant at $38 w+3 d$ gestation, whose birth weight was $3300 \mathrm{~g}$ and length was $50 \mathrm{~cm}$.

\section{Case 2}

A 26-year-old, gravida 1, para 0, pregnant woman underwent ultrasound examination at 12 weeks and 26 weeks of gestation, which presented increased nuchal translucency and fetal pyelic separation in the fetus, separately. Afterwards, the woman underwent amniocentesis for cytogenetic analysis and CMA detection. The karyotype of the fetus was identified as 46,XY. However, CMA detected a $157 \mathrm{~kb}$ duplication in the region of 19q13.42. The couple declined to accept CMA to testify the origin of the 19q duplication and continued the pregnancy. The pregnant woman finally delivered a male infant at $38 w+6 d$ gestation, whose birth weight was $3800 \mathrm{~g}$ and length was $50 \mathrm{~cm}$.

\section{Case 3}

A 29-year-old, gravida 1, para 0, pregnant woman underwent amniocentesis for cytogenetic analysis and CMA detection due to the high risk of maternal serum screening for Down syndrome. No ultrasound findings were 
observed at 21 weeks of gestation. The karyotype of the fetus was 46,XX. Then the CMA detected a $1.445 \mathrm{Mb}$ duplication in the region of $19 q 13.42 q 13.43$. The couple refused the CMA to confirm the chromosomal origin of the fetus and continued the pregnancy. The pregnant woman finally delivered a female infant at $39 w+2 d$ gestation, whose birth weight was $3300 \mathrm{~g}$ and length was $50 \mathrm{~cm}$.

We made a follow up on the postnatal health conditions for all cases, including congenital defects, developmental retardation, body stature, craniofacial dysmorphisms, and skeletal anomalies. All children were in healthy conditions, and no apparent abnormalities were observed till now, but long term follow up analysis was still necessary.

\section{Discussion and conclusions}

In our study, we described three rare prenatal cases with pure $19 \mathrm{q}$ microduplications involving $19 \mathrm{q} 13.42$, ranging from $147 \mathrm{~kb}$ to $1.445 \mathrm{Mb}$. Only in case 2 was possible to study the origin of the anomaly and was proved to be inherited from the healthy mother. Currently, there is a lack of prenatal manifestations about this chromosomal microscopic imbalance. To the best of our knowledge, just five cases with 19q duplication were prenatally detected, presenting prenatal phenotypes including heart defect, anomalies of urinary tract, abnormal nuchal translucency/fold, intrauterine growth restriction and so on $[3,4,12-14]$. Only one case was involved in 19q microduplication [14]. In our study, the prenatally detected 19q duplicated loci were different from the cases mentioned above.

Trisomy 19q, could be regarded as a recognizable syndrome and associated with a wide spectrum of clinical phenotypes, including growth and psychomotor retardation, intellectual disability, low birth weight, microcephaly, short neck, heart malformations, skeletal anomalies, genitourinary anomalies, gastrointestinal defects, seizures and facial dysmorphisms (receding forehead, ptosis, hypertelorism, flat nasal bridge, small nose, short philtrum, down turned mouth, ear anomalies) [4, 12, 15]. Most trisomy 19q cases also carry monosomy of another chromosome, which makes it difficult to establish a clear phenotype-genotype correlation. Till now, only 19q12q13.2 duplication is recognized as an obesity-related syndrome with intellectual disability and minor facial findings [16].

Pure 19q duplications, as a rare chromosomal anomaly, can be usually discovered in live-borns by molecular genetic technique [17]. Till now, there have been limited reports on comparable 19q13.42q13.43 microduplications. To better interpret the genotype-phenotype correlation of this region, we made a summary on clinic data in postnatal and prenatal cases with pure 19q13.42q13.43 duplication, as shown in Table $1[14,18-20]$. The age of these cases ranged from neonate to 8 years. All $19 q$ microduplications were identified through molecular cytogenetic techniques, which varied in size, ranging from $25 \mathrm{~kb}$ to $1.445 \mathrm{Mb}$. Among these duplications, 2/7 cases were de novo, $3 / 7$ cases were parentally inherited, and $2 / 7$ cases were not available. $3 / 7$ cases were postnatally confirmed. Among them, rare anomalies, such as systemic-onset juvenile idiopathic arthritis, Duane retraction syndrome type III and autism spectrum disorder, were observed. 4/7 cases were prenatally detected. Two cases presented abnormal ultrasound findings, including intrauterine growth restriction, fetal pyelic separation and increased nuchal translucency. And the other two cases showed no ultrasound anomalies during the pregnancies. It seemed that the prenatal trisomy 19q13.42q13.43 cases might exhibit normal or abnormal ultrasound findings. Although the infants (cases 1 to 3 ) are now in healthy state, long term follow up analysis is still necessary to confirm whether there will be other emerging clinical symptoms or developmental-behavioral disorders. Among the 3/7 cases with parental inheritance, no abnormal parental phenotypes were observed. The incomplete penetrance might explain why the $19 \mathrm{q}$ duplications were occasionally transmitted through unaffected parents. In addition, these 19q13.42q13.43 duplications encompassed different functional genes, which probably explained the phenotypic diversities in these patients. Generally speaking, the clinic presentations of 19q13.42q13.43 duplication were diverse and atypical, and more evidence should be accumulated to investigate the genotype-phenotype correlation.

In addition, we also made detailed comparisons of cases harboring 19q13.42 microduplication in DECIPHER and ISCA databases to elaborate diverse clinical phenotypes (Fig. 1). Six cases overlapping similar duplications with cases 1 and 2 were recorded. The proportions of pathogenicity were as follows: likely pathogenic $(1 / 6)$ and uncertain (5/6). The incidence rate of clinical features was as follows: Development delay (3/6), intellectual disability (3/6), and autism (2/6). Seven cases shared similar duplication with case 3 in the databases. The clinic pathogenicity in this duplicated locus was uncertain. Only one patient nssv 13649225 presented failure to thrive, hemolytic anemia, short stature and spherocytosis, and no clinic phenotypes were observed for the other six cases. Generally speaking, more evidence should be accumulated to explore the pathogenicity of 19q13.42 microduplication.

The CNVs detected in our case 1 and case 2 shared similar 19q13.42 microduplication, encompassing part NLRP12 gene (OMIM 609648; chr19: 5416521854321990). NLRP12 gene, also known as RNO, PYPAF7, 


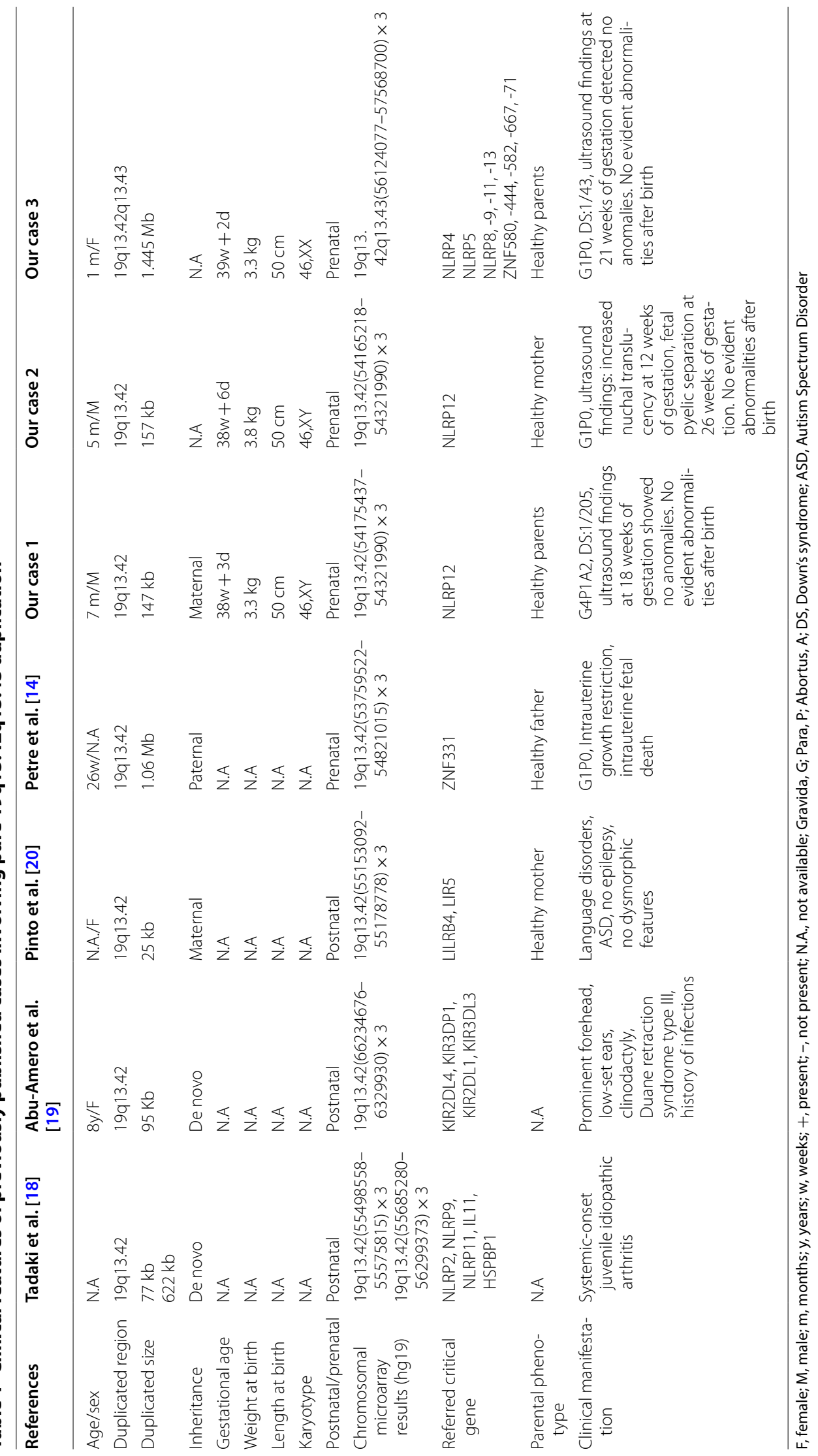




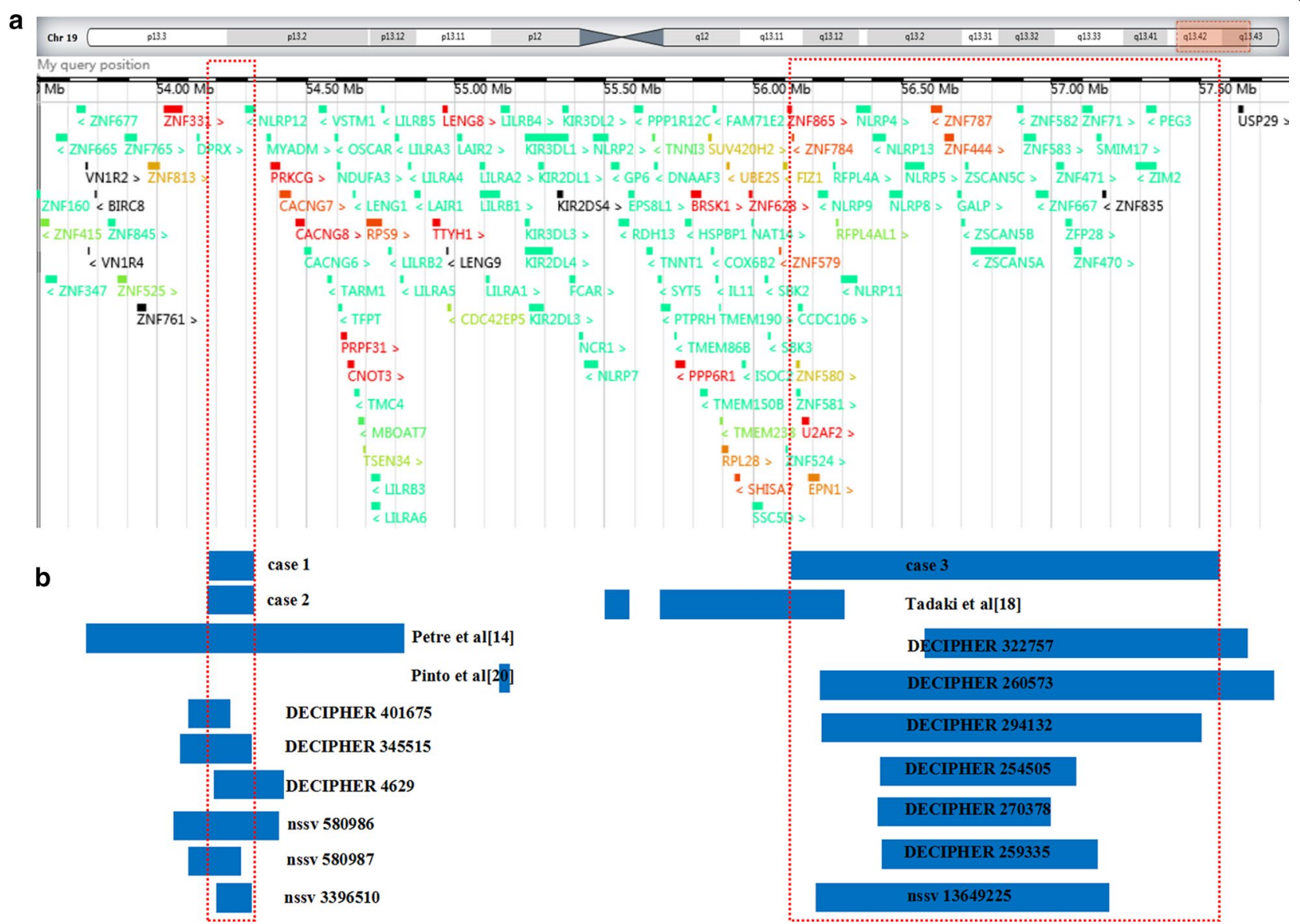

Fig. 1 Scale representation of the duplicated region in the long arm of chromosome 19q13.42q13.43 (https://decipher.sanger.ac.uk/). a Location of genes in the region. $\mathbf{b}$ Microduplications detected in the present cases (cases 1 to 3 ) and previously reported microduplications involving 19q13.42q13.43 in the literature and public databases

and Monarch-1, encodes the protein of NLRP superfamily, which is implicated in the activation of proinflammatory caspases and hyperproduction of interleukin-1 $\beta$ [21]. $N L R P 12$ plays critical roles in the regulation of NF- $\mathrm{KB}$ signaling, inflammasome activation, dendritic cell migration, and transcription of MHC class I genes [22]. The mutations in NLRP12 have been associated with familial cold autoinflammatory syndrome-2 (FCAS2; OMIM 611762), which displays autosomal dominant inheritance. This syndrome can be induced after exposure to cold and characterized by skin urticaria, arthralgia, conjunctivitis, musculoskeletal symptoms, deafness, lymphadenopathy, and abdominal pain, most of which are accompanied by recurrent fever and serologic evidence of inflammation [23, 24]. As is known, dosage-sensitive genes with genome alteration could result in phenotypic effects and be associated with human diseases, including heart disease, cancers, diabetes, neuropsychiatric disorders and others [25]. According to the literature review and database searching, there is no available pathogenic evidence for triplosensitivity associated with NLRP12. However, Galozzi et al. [24] proposed that evolutionary duplication of this gene can have a causative role in autoinflammatory diseases development. Hence, we suggested that these two infants should be followed up regularly on growth and health conditions, especially for autoinflammatory diseases.

For case 3, the detected 19q13.42q13.43 duplication contains 19 OMIM genes. This locus comprises several NLRP (Nucleotide-binding oligomeriztion domain, Leucine rich Repeat and Pyrin domain) family members. NLRP4 is mainly responsible for the inhibition of NF- $\mathrm{B}$ signaling, negative regulation of RLR signaling, autophagy inhibition. NLRP5 is related to the regulation of caspase activation, apoptosis in injured neurons, and embryonic development. The functions of other members (NLRP8, -9, -11, -13) are still to be further investigated $[18,22]$. As is known, the zinc finger proteins (ZNFs) are the largest transcription factor family in human genome, which contain 
finger-like protrusions and play critical roles in physiological and pathophysiological mechanisms [26]. Previous research showed that duplications of zinc finger genes commonly occurred during the evolution [27]. The duplicated locus also included ZNF580, -444, -582, $-667,-71$. Research on the functions of these genes are rare. According to OMIM database, the variations in ZNF582 gene might be associated with intellectual disability. ZNF580 is supposed to be involved in endothelial cell proliferation and migration. Enhancing expression of ZNF667 can inhibit the apoptosis via inhibiting Bax and Fas expression. And ZNF667 might be a new oncogene in human hepatocellular carcinoma as a new therapeutic target through enhancing $B C L-2$ and decreasing $B A X$ expression. Till now, the duplication of these genes could hardly result in severe outcomes in clinic.

Since the young ages of our subjects and the lacking of comparable individuals limit the assessment of growth and development in future, long-term follow up analysis should be guaranteed. In addition, phenotypic diversity, incomplete penetrance, and the inheritance might also be associated with the clinic pathogenicity of $19 q$ microduplication to different degrees, and the clinic data should be further collected.

In our study, we described three rare prenatal cases consisting of $19 \mathrm{q}$ microduplication, encompassing $19 q 13.42$ locus. Our findings would enrich the phenotypic spectrums of $19 \mathrm{q}$ duplication. It is believed that more trisomy $19 \mathrm{q}$ cases with clinic manifestations and molecular genetic characterization would further help to refine clear genotype-phenotype correlations. For prenatally detected $19 \mathrm{q}$ duplications without evident abnormalities, long term follow up should be guaranteed to confirm whether there will be other clinical symptoms or developmental-behavioral disorders afterwards.

\begin{abstract}
Abbreviations
CMA: Chromosomal microarray analysis; CNVs: Copy number variants; DGV Database of Genomic Variants; ISCN 2016: International System for Human Cytogenetic Nomenclature; NCBI: National Center for Biotechnology Information; OMIM: Online Mendelian Inheritance in Man.
\end{abstract}

\section{Acknowledgements}

We express our sincere gratitude to all the staff of the Genetics Laboratory and Andrology Laboratory for their excellent work.

\section{Authors' contributions}

XZ wrote the first draft of the manuscript. FY and QS collected the data of all the fetuses and couples. YJ, JH and LL participated in analysis and interpretation of data. RL reviewed the manuscript and were involved in its critical revision before submission. All authors read and approved the final manuscript.

\section{Funding}

This work was supported by the 10th Youth Foundation of the First Hospital of Jilin University (Grant Number: JDYY102019038), and the Finance
Department Health Special Project of Jilin Province, China (Grant Number: JLSCZD2019-022).

\section{Availability of data and materials}

The data and material used or analysed during the current study are available from the corresponding author on reasonable request.

\section{Ethics approval and consent to participate}

This report was approved by the Ethics Committee of the First Hospital of Jilin University (No. 2019-299). The patients provided written informed consent for participating in this study.

\section{Consent for publication}

Written informed consents were obtained from couples for publication of this manuscript.

\section{Competing interests}

The authors declare that they have no competing interest.

\section{Author details}

${ }^{1}$ Center for Reproductive Medicine, Center for Prenatal Diagnosis, First Hospital, Jilin University, Changchun 130021, China. ${ }^{2}$ Jilin Engineering Research Center for Reproductive Medicine and Genetics, Jilin University, Changchun 130021, China.

Received: 18 November 2020 Accepted: 28 December 2020 Published online: 15 January 2021

\section{References}

1. Lange M, Alfi OS. Trisomy 19 q. Ann Genet. 1976;19(1):17-21.

2. Qorri M, Oei P, Dockery H, McGaughran J. A rare case of a de novo dup(19q) associated with a mild phenotype. J Med Genet. 2002;39(10):E61.

3. Cotter PD, McCurdy LD, Gershin IF, Babu A, Willner JP, Desnick RJ. Prenatal detection and molecular characterization of a de novo duplication of the distal long arm of chromosome 19. Am J Med Genet. 1997;71(3):325-8.

4. Rombout S, Sartenaer D, Parmentier B, Dugauquier C, Gillerot Y. A rare case of de novo distal 19q trisomy prenatally diagnosed. Prenat Diagn. 2004;24(10):822-7.

5. Resta N, De Cosmo L, Susca FC, Capodiferro D, Nardone AM, Pastorivo $D$, et al. De novo unbalanced translocation leading to monosomy 9p24.3p24.1 and trisomy 19q13.42q13.43 characterized by microarraybased comparative genomic hybridization in a child with partial cortical dysplasia and craniofacial dysmorphisms without trigonocephaly. Am J Med Genet A. 2013;161A(3):632-6.

6. Nacinovich R, Villa N, Broggi F, Tavaniello C, Bomba M, Conconi D, et al. 19q12q13.2 duplication syndrome: neuropsychiatric long-term follow-up of a new case and literature update. Neuropsychiatr Dis Treat. 2017:13:2545-50.

7. Schluth-Bolard C, Till M, Rafat A, Labalme A, Le Lorc'h M, Banquart E, et al. Monosomy 19pter and trisomy 19q13-qter in two siblings arising from a maternal pericentric inversion: clinical data and molecular characterization. Eur J Med Genet. 2008:51(6):622-30.

8. López-Exposito I, Guillén-Navarro E, Bafallíu JA, Bernabé MC, Escalona A, Fuster C. Duplication 19q13-qter and deletion 19p13-pter arising from an inversion (19)(p13.3q13.3) of maternal origin. Eur J Med Genet. 2006;49(6):511-5.

9. Wapner RJ, Martin CL, Levy B, Ballif BC, Eng CM, Zachary JM, et al. Chromosomal microarray versus karyotyping for prenatal diagnosis. N Engl J Med. 2012;367(23):2175-84

10. McGowan-Jordan J, Simons A, Schmid M. An international system for human cytogenomic nomenclature, S. Karger, Ed., Karger, Basel, 2016.

11. Zhang H, Liu X, Geng D, Yue F, Jiang Y, Liu R, et al. Molecular cytogenetic characterization of a mosaic small supernumerary marker chromosome derived from chromosome $Y$ in an azoospermic male: a case report. Medicine. 2019:98(30):e16661.

12. Tercanli S, Hösli I, Berlinger A, Beyer R, Achermann J, Holzgreve W. Prenatal diagnosis of a partial trisomy 19q. Prenat Diagn. 2000;20(8):663-5. 
13. Babić I, Brajenović-Milić B, Petrović O, Mustać E, Kapović M. Prenatal diagnosis of complete trisomy 19q. Prenat Diagn. 2007;27(7):644-7.

14. Petre G, Lorès P, Sartelet H, Truffot A, Poreau B, Brandeis S, et al. Genomic duplication in the 19q13.42 imprinted region identified as a new genetic cause of intrauterine growth restriction. Clin Genet. 2018;94(6):575-80.

15. Sauter SM, Böhm D, Bartels I, Burfeind P, Laccone FA, Neesen J, et al. Partial trisomy of distal $19 \mathrm{q}$ detected by quantitative real-time PCR and FISH in a girl with mild facial dysmorphism, hypotonia and developmental delay. Am J Med Genet A. 2007:143A(10):1091-9.

16. Nevado J, Mergener R, Palomares-Bralo M, Souza KR, Vallespín E, Mena R, et al. New microdeletion and microduplication syndromes: a comprehensive review. Genet Mol Biol. 2014;37(1 Suppl):210-9.

17. Palomares Bralo M, Delicado A, Lapunzina P, Velázquez Fragua R, Villa O, Angeles Mori M, et al. Direct tandem duplication in chromosome 19q characterized by array CGH. Eur J Med Genet. 2008;51(3):257-63.

18. Tadaki H, Saitsu H, Nishimura-Tadaki A, Imagawa T, Kikuchi M, Hara R, et al. De novo 19q13.42 duplications involving NLRP gene cluster in a patient with systemic-onset juvenile idiopathic arthritis. J Hum Genet. 2011;56(5):343-7.

19. Abu-Amero KK, Kondkar AA, Al Otaibi A, Alorainy IA, Khan AO, Hellani AM et al. Partial duplication of chromosome 19 associated with syndromic duane retraction syndrome. Ophthalmic Genet. 2015;36(1):14-20.

20. Pinto D, Pagnamenta AT, Klei L, Anney R, Merico D, Regan R, et al. Functional impact of global rare copy number variation in autism spectrum disorders. Nature. 2010;466(7304):368-72.
21. Kostik MM, Suspitsin EN, Guseva MN, Levina AS, Kazantseva AY, Sokolenko $A P$, et al. Multigene sequencing reveals heterogeneity of NLRP12-related autoinflammatory disorders. Rheumatol Int. 2018;38(5):887-93.

22. Tuncer S, Fiorillo MT, Sorrentino R. The multifaceted nature of NLRP12. J Leukoc Biol. 2014:96(6):991-1000.

23. Hull KM, Shoham N, Chae JJ, Aksentijevich I, Kastner DL. The expanding spectrum of systemic autoinflammatory disorders and their rheumatic manifestations. Curr Opin Rheumatol. 2003;15(1):61-9.

24. Galozzi P, Punzi L, Sfriso P. Clinical overlapping in autoinflammatory diseases: the role of gene duplication. Front Immunol. 2017;8:392.

25. Rice AM, McLysaght A. Dosage-sensitive genes in evolution and disease. BMC Biol. 2017;15(1):78

26. Squassina A, Meloni A, Chillotti C, Pisanu C. Zinc finger proteins in psychiatric disorders and response to psychotropic medications. Psychiatr Genet. 2019;29(5):132-41

27. Looman C, Abrink M, Mark C, Hellman L. KRAB zinc finger proteins: an analysis of the molecular mechanisms governing their increase in numbers and complexity during evolution. Mol Biol Evol. 2002;19(12):2118-30

\section{Publisher's Note}

Springer Nature remains neutral with regard to jurisdictional claims in published maps and institutional affiliations.
Ready to submit your research? Choose BMC and benefit from:

- fast, convenient online submission

- thorough peer review by experienced researchers in your field

- rapid publication on acceptance

- support for research data, including large and complex data types

- gold Open Access which fosters wider collaboration and increased citations

- maximum visibility for your research: over $100 \mathrm{M}$ website views per year

At BMC, research is always in progress.

Learn more biomedcentral.com/submissions 